\title{
Medical Child Abuse: When Parents Harm Their Children
}

Ami Rokach

Dept. of Psychology, York University, Toronto, Canada

*Corresponding Author: Ami Rokach, Dept. of Psychology, York University, Toronto, Canada. E-mail: arokach@yorku.ca Received Date: September 28, 2020; Accepted Date: October 09, 2020; Published Date; October 14, 2020.

Citation: Ami Rokach, Medical Child Abuse: When Parents Harm Their Children, J. Psychology and Mental Health Care. 4(5). Doi: 10.31579/2637-8892/102.

Copyright: () 2020 Ami Rokac, This is an open-access article distributed under the terms of the Creative Commons Attribution License, which permits unrestricted use, distribution, and reproduction in any medium,provided the original author and source are credited

\section{Abstract}

This brief review of medical child abuse and treatment approaches is meant to highlight a problem which gains in frequency lately. That of a parent, usually, who is expected to love and care for the child actually harming that child in ways that may result in many medical examinations, painful invasive procedures, and even unnecessary surgeries.

Key words: child abuse; medical child abuse; mothers; parent; perpetrators; treatment

\section{Medical child abuse: When parents harm their children}

Edward [not his real name] came to see me due to his incredibly strong fear of physicians and hospitals. He was not; initially; aware of the cause of that very intense fear. As we started to explore his history and childhood; he spoke of having been a sick child; who was frequently hospitalized; underwent many invasive and painful medical procedures; with his mother always by his side; caring deeply for him; deeply involved in his treatment and constantly begging physicians and nurses to help her son. As his illness and treatment regimen continued to be explored; it became clear that at home; his diet was very limited; he was constantly hungry; and at times his mother would give him "some pills" which he was told are to enhance his health; but which ended up causing him painful sores in his intestines which not only resulted in rectal bleeding; but later developed into internal bleeding which necessitated surgery. Complaining; at home; about his discomfort and pain; the mother would take him to the ER; and demand that her beloved son be attended to immediately. It took many months to decipher what happened. The mother; who was herself abused as a child; had started to give Edward pills that she was prescribed to treat menstrual pain. These harmed Edward's intestines and resulted in his hospitalization and painful treatment.

"Medical child abuse is defined as a child receiving unnecessary and harmful or potentially harmful medical care at the instigation of a caretaker... It results from a parent relating to a physician in a way that leads the medical care provider to expose the child to possibly harmful treatment. Any medical procedure; for example; a blood draw; or a trial of medication that is potentially harmful; could be considered abusive if there was no clear medical reason for it to happen" (Isaac \& Roesler; 2010; p. 291).

Coming to make a diagnosis of medical child abuse; it is essential to understand whether the child received harmful medical care based on a parent's actions. Medical child abuse; part of the general term child maltreatment; has several characteristics that distinguish it from the other types of child abuse. For instance; when the child is presented in the hospital; the physician does not have to know why did the parent harm the child; nor what his motivation is. Medical treatment is the first priority
(Isaac et al.; 2016; Stirling; 2007). While medical neglect occurs when a parent does not provide the medical care that the child needs; medical abuse is reserved for a parent who is seeking excessive treatment for the child's medical issues which have been created intentionally by the parent. Beating; burning; biting; or cutting is termed physical abuse. Psychological abuse is related to belittling; criticism; shaming; or blaming. Likewise; medical abuse may have various manifestations. Abuse may range from mild to severe. If; for example; a mother taking the child for various unnecessary medical appointments; he thus misses school and falls behind his classmates would be on the mild end of the continuum; while a child undergoing unnecessary surgery is at the extreme end. Long-term effects of medical abuse have not been studied as well as other forms of child maltreatment; but we do know that the effects of medical child abuse are consistent with other forms of abuse (Isaac \& Roesler; 2010; Libow; 1995).

There are several phases of intervention regarding all kinds of abuse; and they pertain to medical abuse as well: firstly; we need to establish that abuse is; indeed; taking place. Then we need to find a way to stop it. After it is stopped; the child needs to be continuously safe; and then the physical and/or psychological effects of the abuse need to be attended to.

The goal is to accomplish all this while both protecting the integrity of the family. Just as the forms of child maltreatment share many similarities we can find similarities between the various perpetrators as well. Research shows that perpetrators had unhappy childhoods with histories of maltreatment. And while it is not a forgone conclusion that anyone what was maltreated will abuse their own children; people commonly treat their children the way they were treated by their caregivers while growing up. Perpetrators of all forms of abuse often lie about their involvement and try to justify what they cannot hide. In general; they do not take responsibility to what was done to the child. The main difference between physical; sexual and medical abuse is by the type of harm caused to the child. In medical abuse; the child received unnecessary and at times painful medical treatment (Isaac \& Roesler; 2010).

There are many ways that children can be victimized while by receiving excessive medical care. Unnecessary treatment may be performed on any organ and symptom presentation is limited only to the perpetrator's 
sophistication and imagination. Organic conditions that may be mimicked by the actions of the perpetrator include:

“• Exaggeration: i.e. embellishing or heightening an existing medical condition or symptom in order to obtain more medical attention and care.

- Fabrication: i.e. lying about non-existent symptoms.

- Persuasion: i.e. repeatedly demanding medical care until the physician gives in to the demand; altering perceptions of a child's condition.

- Simulation: i.e. contaminating specimens collected for laboratory analysis.

- Induction: i.e. actually directly hurting the child and then seeking unnecessary medical treatment for the induced condition" (Isaac \& Roesler; 2010; p. 299).

To illustrate; apnea may be produced by manual suffocation of the child; poisoning; or induce hypoglycemia; seizures may be brought about by suffocation or poisoning; laxative poisoning; salt poisoning or contamination may result in diarrhea; CNS depression may be caused by drugs or suffocation; or a rash cold be brought about by the child ingesting drugs or caustics (Carter; Izsak; \& Marlow; 2006; Holstege \& Dobmeier; 2006).

\section{Prevalence of medical abuse}

Child abuse is not; by itself; an illness but may result in one or have psychological consequences. It is an act which is intentionally committed by an adult; and in order to know its prevalence; a consideration of several factors is required. The definition; culture that the abuse occurs; and the degree; mild or severe; are all important indications. Culture influences the definition of abuse; or whether a behavior is considered abuse at all. The mild medical abuse may be quite frequent; while the severe one is; fortunately; rare. McClure and colleagues (McClure; Davis; Meadow; \& Sibert; 1996) searched child protection records in England and discovered medical abuse which mainly involved induction of illness such as suffocation and non-accidental poisoning. Based on their research the authors estimated a prevalence rate of 0.5 children in 100;000 children. In New Zealand research indicated that 2 in 100;000 children met their criteria for medical abuse (Denny; Grant; \& Pinnock; 2001).

\section{Motivation of the perpetrator}

The motivation and reason why the perpetrator committed the medical abuse is varied. They may include rage at the child victim or at the healthcare provider; a component of help seeking; a delusion that the child is actually suffering form a medical condition that needs intervention; and commonly involves secondary gains (Levin \& Sheridan; 1995). In a true case of medical abuse; the perpetrators may try to explain the situation with reasons that do not conform with the reality of the situation. It is; actually; an interesting question as to why did a perpetrator decide to harm the child in that way. If we examine sexual abuse; or emotional abuse it may be easier to explain than understanding why a parent would put the child in harms way by subjecting the child to unnecessary medical treatments. The concern has less to do with the perpetrator than with medical professionals. The concern with motivation of perpetrators of MCA may reflect communitywide countertransference on the part of medical care providers; who may feel angry; betrayed and seem sometime foolish for carrying out medical procedures against the child's best interests (Isaac \& Roesler; 2010).

\section{Characteristics of perpetrators of MCA}

Although cases of FDIA [Factitious Disorder Imposed by Another] have been described in the literature since 1977 (Meadow; 1977); the characteristics of these perpetrators still remain unclear. Since covert sabotage is inherent in these cases; it may take a long time for the medical personnel to identify the abusive adult; usually the mother. During this time; the child continues to be subjected to repeated painful and invasive medical procedures; which can be detrimental to their long-term health and life (Day et al.; 2017). It is estimated that $100 \%$ of victimized children will suffer short-term illnesses (Rosenberg; 1987). 8\% of this population will continue to suffer from long-term illnesses; ultimately leading to death $9 \%$ of the time (Rosenberg; 1987). Consequently; being able to; hopefully quickly; identify the perpetrator is highly important. While there is not much research regarding the personality structure of perpetrators; there are suggestions as to what is involved in FDIA; based on clinical syndromes and personality disorders. We know that mothers are more likely to be engaged in MCA than fathers (Schreier et al.; 1994). The mother; then; is often described by the healthcare providers as being cooperative; loving; and attentive to her child - showing reluctance to leave their bedside; and remains in the hospital as much as she can; sometimes even living there (Fischler; 1983; Meadow; 1982). These mothers tend to develop close; personal relationships with hospital staff and become close with parents of other ill children and offer them; voluntarily; emotional support (Parnell \& Day; 1998). A majority of mother-perpetrators usually have extensive knowledge pertaining to medical terminology; diagnoses; and procedures (Day \& Moseley; 2010). During times when their child seems to be in crisis; the perpetrators remain calm and welcome; even encouraging a host of potentially painful medical proceduresfor their child (Hanon; 1991). These mothers may get involved in the child's treatment by managing the child's medication regimen; or post operative care (Day \& Moseley; 2010).

In regards to the mother-perpetrators' personal history; it was observed that she may display signs and symptoms similar to those that she fabricated in her child (Meadow; 1982). The mother may also have a history of Factitious Disorder or actual medical problems varying in degrees of severity (Gray \& Bentovim; 1996; Rosenberg; 1987). Perpetrators report high rates of somatisation disorders; in addition to emotional deprivation; physical abuse and sexual abuse (Bass \& Jones; 2011; Gray \& Bentovim; 1996). Additionally; they have experienced significant grief relating to conception problems; miscarriage; stillbirth or death (Gray \& Bentovim; 1996). The presence of personality disorders among this population have been frequently suggested. Specific personality disorders that were observed in FIDA mothers included borderline; histrionic; dependent; narcissistic; and antisocial along with anxiety and depressive disorders (Bass \& Jones; 2011; Day \& Moseley; 2010; Lacey; et al.; 1993; Meadow; 1994).

\section{Treatment of MCA}

In terms of treatment outcomes; MCA perpetrators were found to be extremely difficult to successfully treat; especially those at the severe end of the spectrum (Berg \& Jones; 1999; Black \& Hollis; 1996; Klepper et al.; 2008). According to research; successful treatment involves the acknowledgement of abusive behaviours and acceptance of responsibility by the perpetrators. This may include the involvement of familial support; treatment for debilitating personality disorders or an emphasis of the individual's personality strengths for succession. Before beginning therapy with the perpetrator; the therapist should assess; as much as possible; the likelihood for success. Some might feel that treatment may only be possible when the parent "confesses"; acknowledges his/her behaviors or is open to treatment; although; most perpetrators are not inclined to do so during the beginning of their therapeutic journey. If therapy begins; and the client is unable to build trust and enter into the treatment in a timely and genuine manner; the therapist may consider ending treatment.

\section{Safety Plan and Plan for Reunification}

It is important to provide clear guidelines regarding expectations on how that will affect any possible reunification. This includes clearly explaining 
to them how they would be evaluated for reunification; and how clinical treatment of the parents and child victim(s) will proceed (Sanders \& Bursch; 2002).

\section{Individual Therapy for Other Family Members or Friends}

There may be situations where the needs of the child dictate that he be placed with other relatives or family friends (Sanders \& Ayoub; 2018). Family members; who may have colluded with the abuser; might also benefit from therapy - as there most likely was some awareness of the abuse happening in the first place. It is imperative that all caretakers are able to acknowledge the abuse and deal with it in healthy ways. Family members may feel shocked; angry; betrayed or guilty for having enabled the abuse; and need a forum to address those feelings (Sanders; 1995).

\section{References}

1. Bass; C.; \& Jones; D. (2011). Psychopathology of perpetrators of fabricated or induced illness in children: Case series. The British Journal of Psychiatry; 199(2); 113 - 118. https://doi.org/10.1192/bjp.bp.109.074088

2. Berg; B.; and Jones; D. (1999). Outcome of psychiatric intervention in factitious illness by proxy (Munchausen's syndrome by proxy). Archives of Disease in Childhood; 81(6); 465 - 472. DOI: 10.1136/adc.81.6.465

3. Black; D.; and Hollis; P. (1996). Psychiatric treatment of factitious illness in an infant (Munchausen by proxy syndrome). Clinical Child Psychology and Psychiatry; 1(1); 89 - 98. DOI: $10.1177 / 1359104596011008$

4. Carter; K. E.; Izsak; E.; \& Marlow; J. (2006). Munchausen syndrome by proxy caused by ipecac poisoning.

5. Pediatric Emergency Care; 22(9); 655-656.

6. Davis; P.; McClure; R.; Rolfe; K.; Chessman; N.; Pearson; S.; et al. (1998). Procedures; placement; and risks of further abuse after Munchausen syndrome by proxy; non-accidental poisoning; and non-accidental suffocation. Archives of Diseases in Childhood; 78(3); 217 - 221. DOI:

10.1136/adc.78.3.217

7. Day; D. O.; and Moseley; R. L. (2010). Munchausen by proxy syndrome. Journal of Forensic Psychology

8. Practice; 10(1); 13 - 36. DOI: 10.1080/15228930903172981

9. Day; L. B.; Faust; J.; Black; R.A.; Day; D.O. and Alexander; A. (2017). Personality profiles of factitious disorder imposed by mothers: A comparative analysis. Journal of Child Custody; 14(2-3); 191-208; DOI: 10.1080/15379418.2017.1331780

10. Denny; S. J.; Grant; C. C.; and Pinnock; R. (2001). Epidemiology of Munchausen syndrome by proxy in New Zealand. Journal of Pediatric Child Health; 37(3); 240-243.

11. Fischler; R. S. (1983). Poisoning: A syndrome of child abuse. American family physician; 28(6); 103.

12. Gray; J.; and Bentovim; A. (1996). Illness induction syndrome: Paper I - A series of 41 children from 37 families identified at the great ormond street hospital for children NHS trust. Child Abuse \& Neglect; 20(8); 655 - 673. DOI: 10.1016/01452134(96)00055-5

13. Hanon; K. (1991). Child abuse: Munchausen syndrome by proxy. FBI Law Enforcement Bulletin; 60(12); 811.

14. Holstege; C. P.; and Dobmeier; S. G. (2006). Criminal poisoning: Munchausen by proxy. Clinics in

15. Laboratory Medicine; 26(1); 243-253.

16. Isaac; R.; Creamer; L. \& Trent; M. (2016). The deconstruction of a complex case of medical child abuse.
17. Open Medicine Journal; 3; (Suppl 2: M5) 202-211.

18. Isaac; R. and Roesler; T.A. (2010). Medical child abuse. In A.P.; Giardino; M.A. Lyn \& E.R. Giardino (Eds.). A practical guide to the evaluation of child physical abuse and neglect (2nd ed.; pp. 291-306). Springer.

19. Klepper; J.; Heringhaus; A.; Wurthmann; C.; \& Volt; T. (2008). Expect the unexpected: Favourable outcome in Munchausen by proxy syndrome. European Journal of Paediatrics; 167(9); 1085 - 1088 DOI: 10.1007/s00431-007-0627-4

20. Lacy; S. R.; Cooper; C.; Runyan; D. K.; and Azizkhan; R. G. (1993). Munchausen syndrome by proxy: Patterns of presentation to pediatric surgeons. Journal of Pediatric Surgery; 28(6); 827 - 832. DOI: 10.1016/0022-3468(93)90337-K

21. Levin; A. V.; and Sheridan; M. S. (Eds.). (1995). Munchausen syndrome by proxy: Issues in diagnosis and treatment. New York: Lexington Books.

22. Libow; J. A. (1995). Munchausen by proxy victims in adulthood: A first look. Child Abuse and Neglect; 19(9); 11311142 .

23. Meadow; R. (1977). Munchausen syndrome by proxy: The hinterland of child abuse. Hospital Practice; 310(8033); $343-$ 345. DOI: 10.1016/S0140-6736(77)91497-0

24. Meadow; R. (1982). Munchausen syndrome by proxy. Archives of Disease in Childhood; 57(2); 92 - 98. DOI: 10.1136/adc.57.2.92

25. Meadow; S. R. (1994). Who's to blame-Mothers; Munchausen or medicine? Journal of the Royal College of Physicians of London; 28(4); 332 - 337.

26. McClure; R. J.; Davis; P. M.; Meadow; S. R.; and Sibert; J. R. (1996). Epidemiology of Munchausen syndrome by proxy; nonaccidental poisoning; and non-accidental suffocation. Archives of Disease in Childhood; 75(1); 57-61.

27. Parnell; T. F.; and Day; D. O. (1998). Munchausen by proxy syndrome: Misunderstood child abuse (1st ed.). SAGE Publications.

28. Rosenberg; D. A. (1987). Web of deceit: A literature review of Munchausen syndrome by proxy. Child Abuse \& Neglect; 11(4); 547 - 563. DOI: 10.1016/0145-2134(87)90081-0

29. Sanders; M. J. (1995). Symptom coaching: Factitious disorder by proxy with older children. Child

30. Psychology Review; 15(5); 423 - 442. DOI: 10.1016/02727358(95)00024-J

31. Sanders; M. J.; \& Bursch; B. (2002). Forensic assessment of illness falsification; Munchausen by proxy; and factitious disorder; NOS. Child Maltreatment; 7(2); 112 - 124. DOI: 10.1177/1077559502007002003

32. Sanders; M. J.; and Ayoub; C. (2018). Munchausen by proxy: Risk assessment; support and treatment of spouses and other family caregivers. APSAC Advisor; 30; 66-75.

33. Sanders; M.J. \& Bursch; B. (2020). Psychological treatment of factitious disorder imposed on another/ Munchausen by Proxy Abuse. Journal of Clinical Psychology in Medical Settings; 27; 139-149. DOI: 10.1007/s10880-019-09630-6

34. Schreier; H. A.; Libow; J. A.; Jellinek; M. S.; and Biederman; J. (1994). Munchausen by proxy syndrome: A clinical fable for our times. Journal of the American Academy of Child \& Adolescent Psychiatry; 33(6); 904 - 905. DOI:10.1097/00004583199407000-00017

35. Stirling; J.; Jr. (2007). Beyond Munchausen syndrome by proxy: Identification and treatment of child abuse in a medical setting. Pediatrics; 119(5); 1026-1030. 